\title{
خطر التعدين في المنطقة الدولية وتأثيره على الحياة البحرية
}

Mining in the international zone and its impact on marine life

تاريخ الاستلام : 2019/11/17؛ تاريخ القبول : 2020/09/06

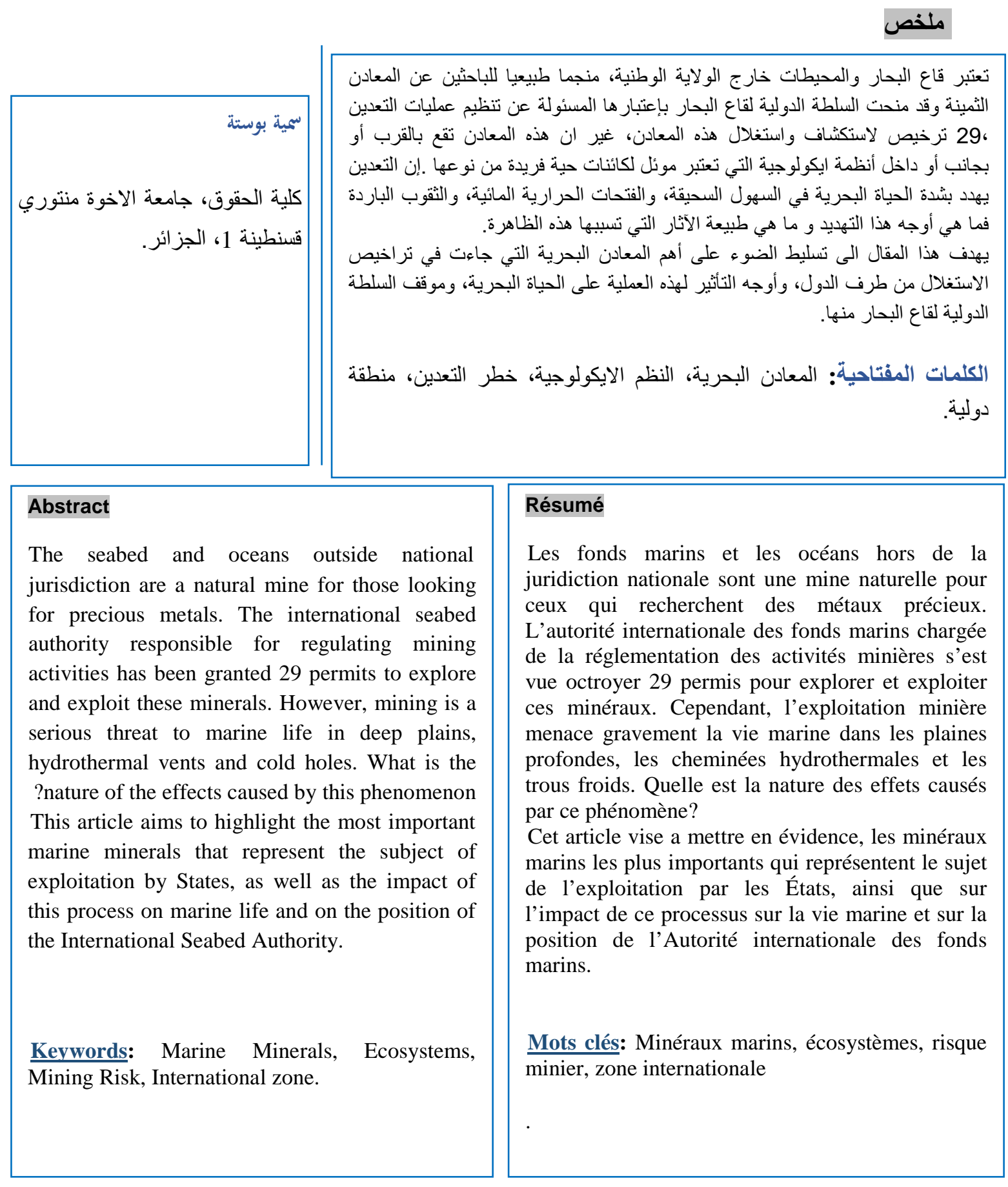

* Corresponding author, e-mail: soumeyaboucetta99@gmail.com 
تتسم قاع البحار والمحيطات بمجموعة متميزة من النظم الإيكولوجية كالقتحات

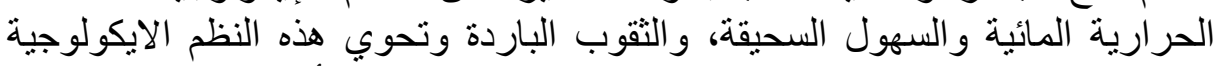

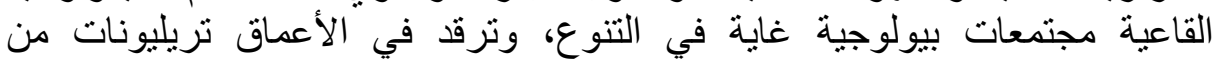

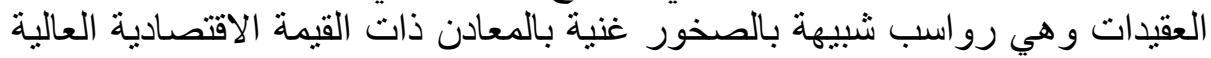

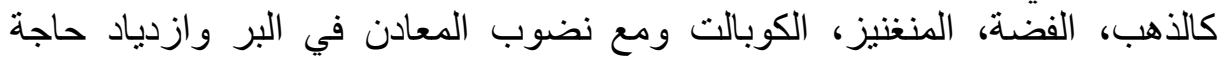

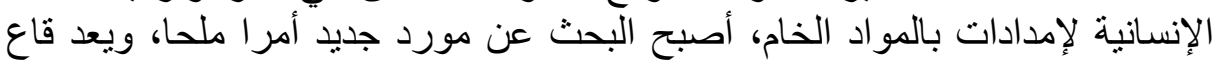

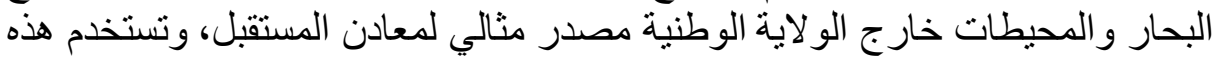

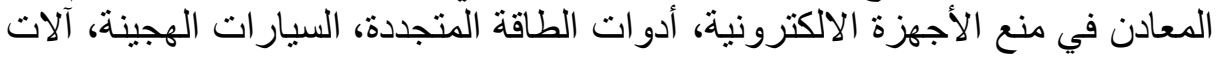

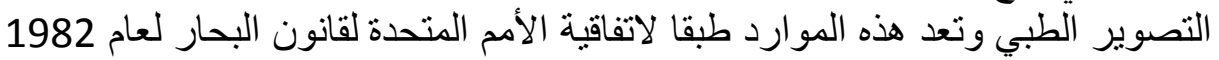

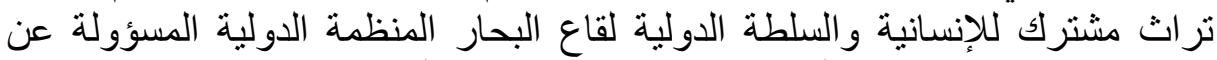

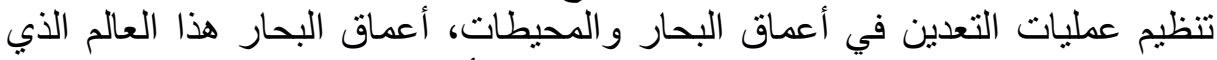

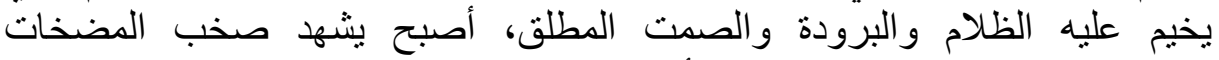

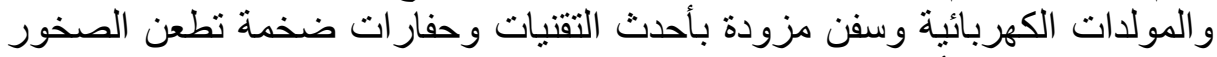

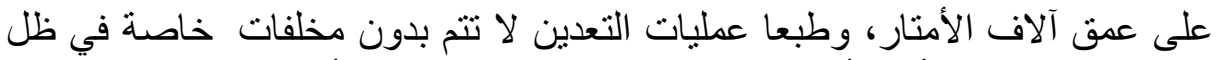

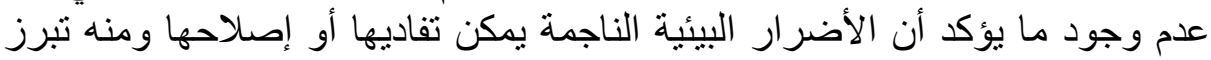

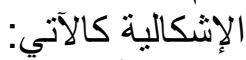
ما هو تأثير المعادن البحرية التي يجري استغلالها في المنطقة الدولية على الحياة

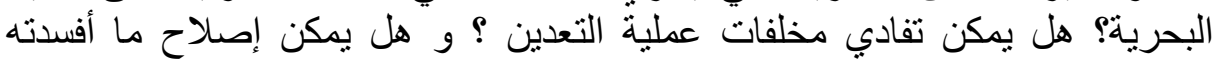

$$
\begin{aligned}
& \text { عمليات التعدين . } \\
& \text { و قد رصدنا لهذه الدراسة فرضيات نوجز هات البهات فيما يلي : }
\end{aligned}
$$

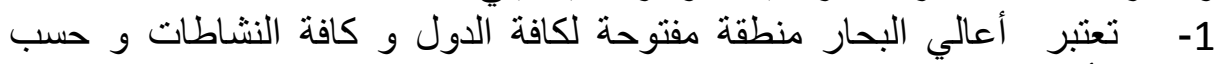

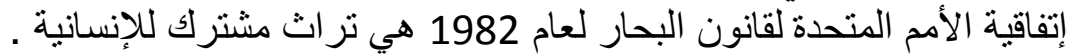

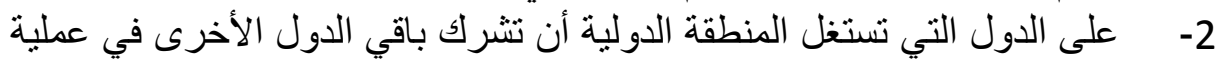

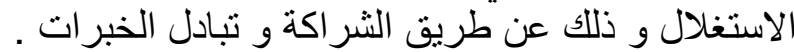

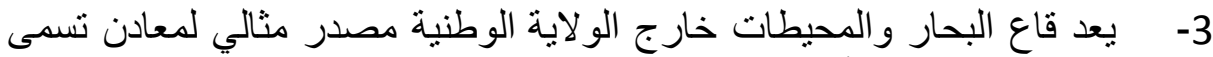

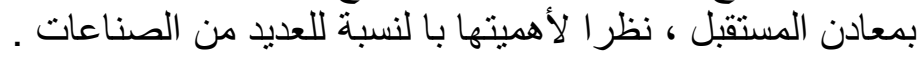

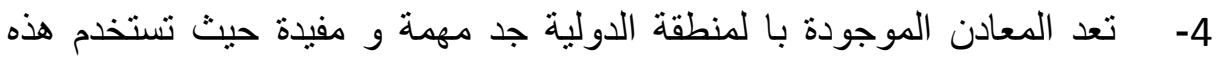

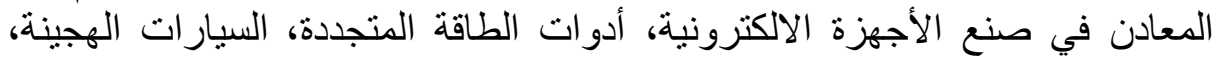
آلات التصوير الطبي و فير الإنغة ها.

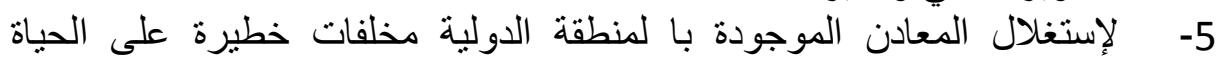

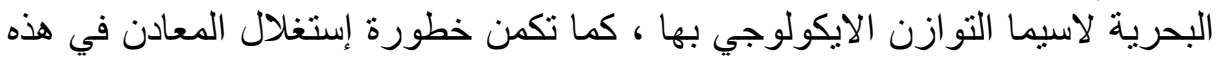

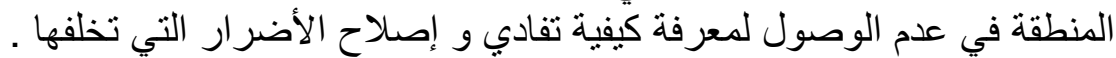

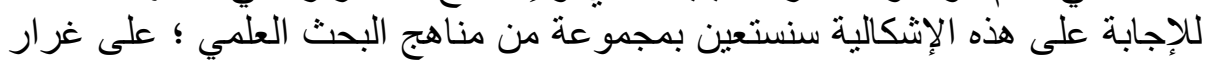

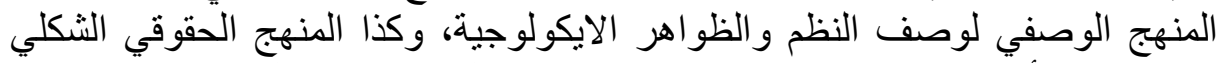

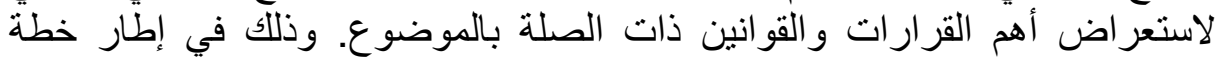

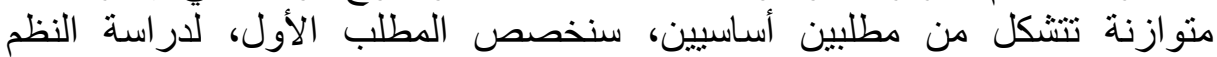

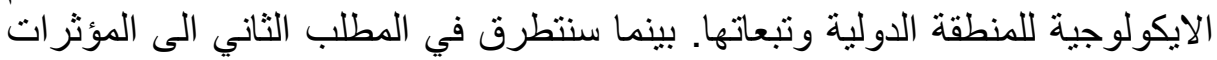

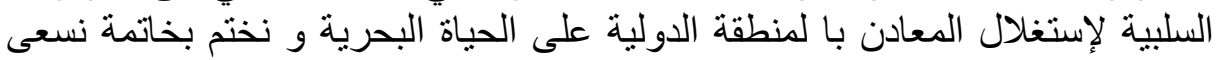

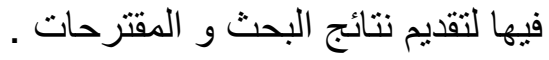


كان الاعتقاد السائد أن قاع البحار و المحيطات عبارة عن صحر اء قاحلة تنعدم فيها

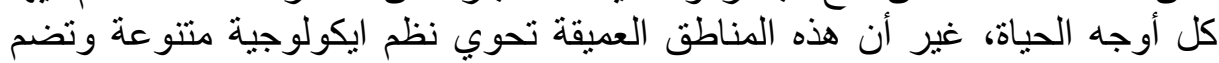

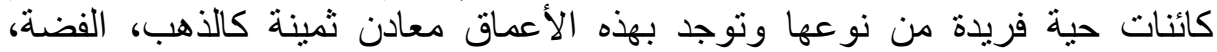

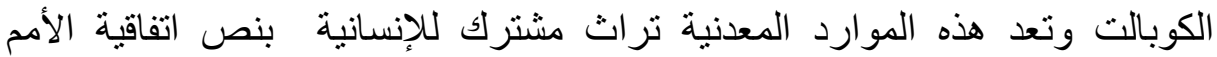

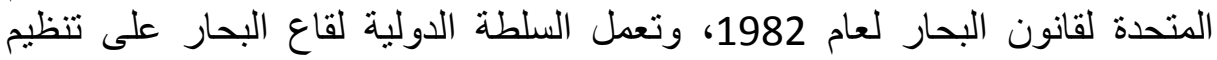

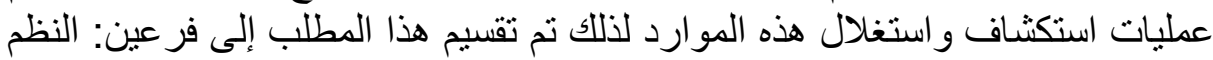

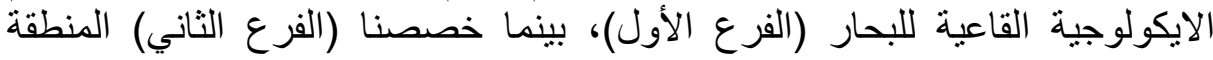
الدولية والسلطة الممارسة عليها.

\section{الفرع الأول: النظم الايكولوجية لقاع للبحار}

يحتوي قاع البحار على الكثير من المظاهر التضاريسية وسوف نتطرق في هذا

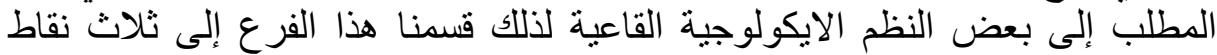

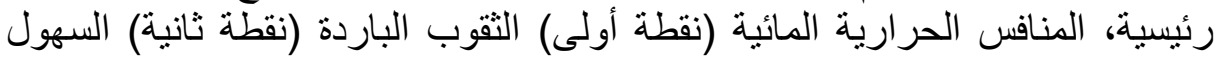
الساحقة (نقطة ثالثة).

\section{أولا: المنافس الحرارية المائية}

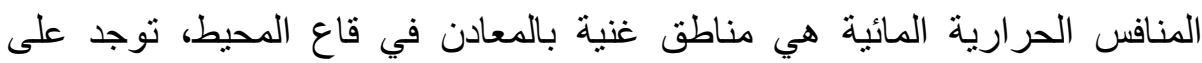

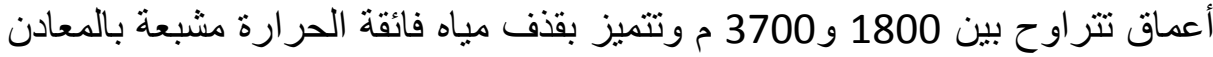

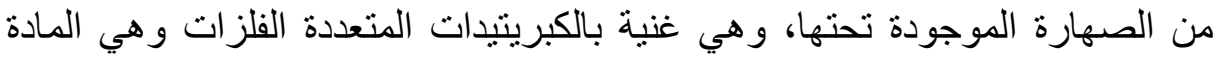

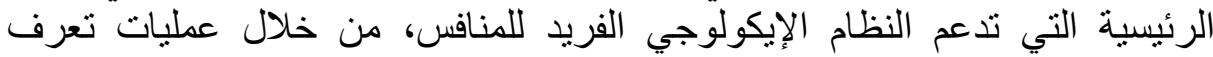

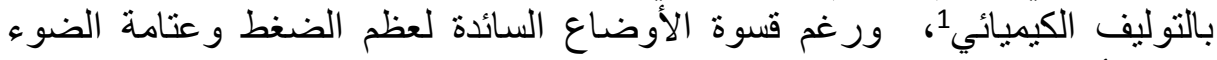

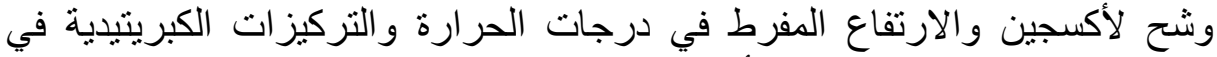

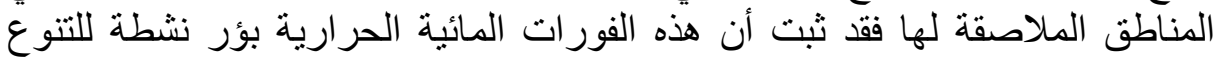

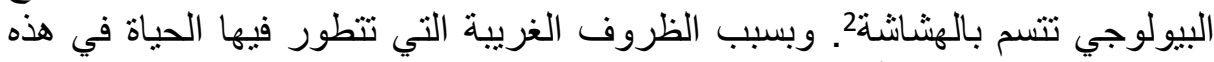

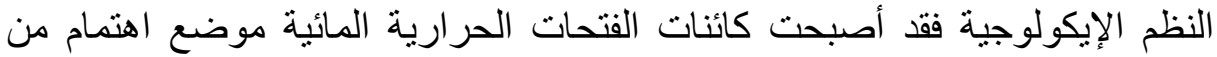

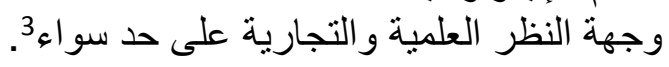

ثانيا: الثقوب الباردة البطرة

الثقوب الباردة هي مسارب يخرج منها الميثان والكبريتيدات عبر الرواسب الكبان المتر اكمة

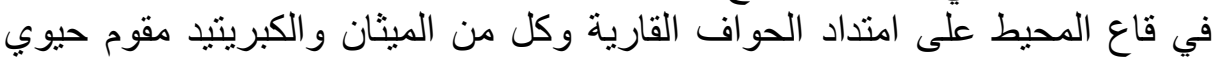

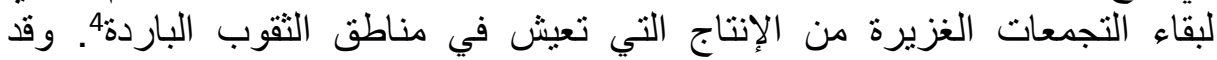

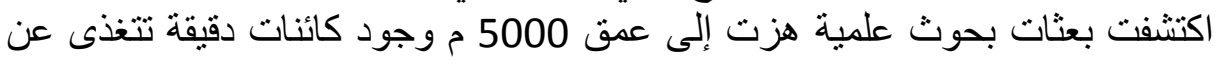

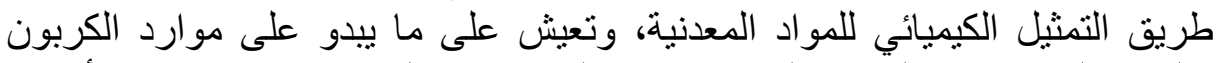

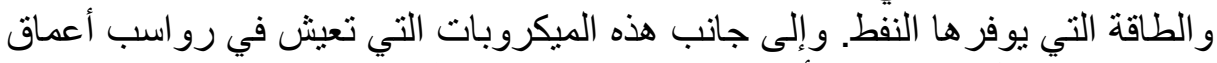
المحيطات تشمل الكائنات الأخرى الموجودة في هذه المناطق البران الديدان الانبوبية، وبلح

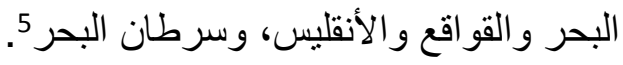

\section{ثالثا: السهول السحيقة}

السهول السحيقة موائل رتيبة من تلال مترامية من الرواسب تعيش فيها أعداد قلبلة

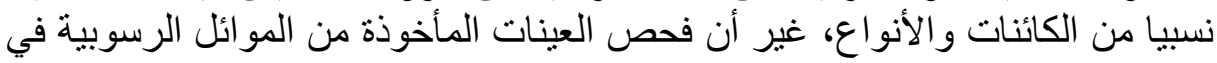


العقود الأربعة الأخيرة يحمل على الظن بأن اللافقاريات التي تعيش في رواسب

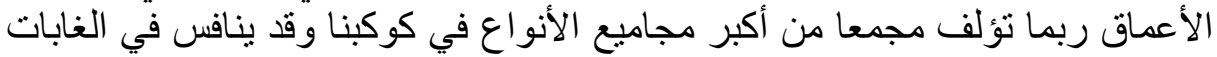

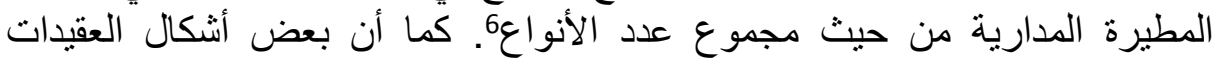

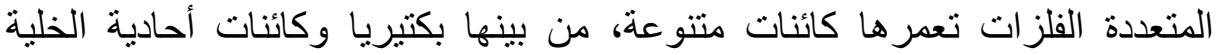

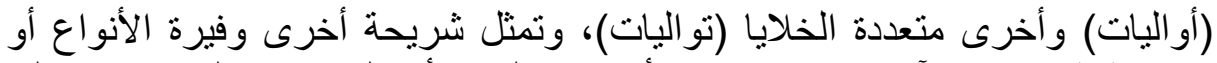

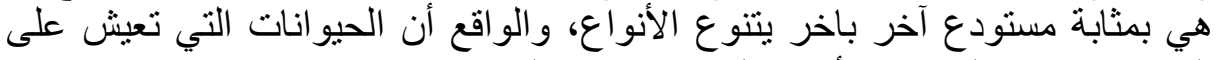

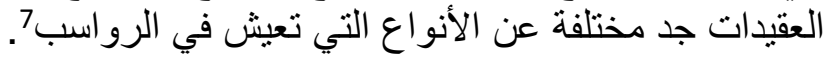

\section{الفرع الثاني: المنطقة الدولية والسلطة الممارسة عليها}

سنتطرق في هذا المطلب إلى تعريف المنطقة الدولية والنتائج المترنبة عن كونها

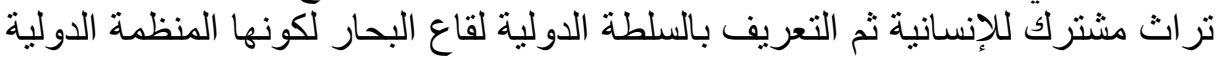

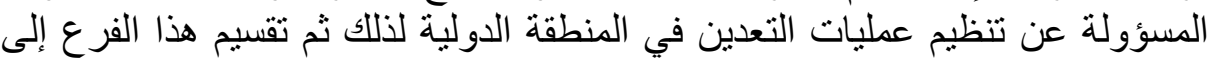

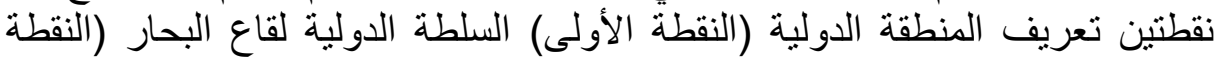

\section{أولا: تعريف المنطقة الدولية}

حددت المادة (1/1) من الاتفاقية العامة للبحار المنطقة الدولية بأنها تعني قاع البحار

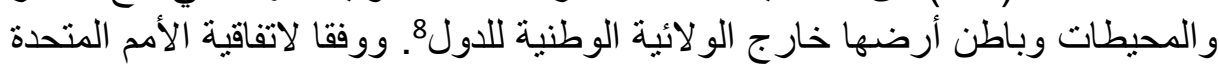

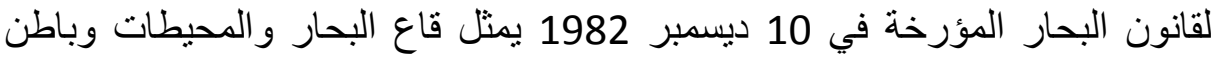
أرضها خارج الولاية الوطنية وكذا مواردها تراث فئرية مشترك للإنسانية ينم استكثافها

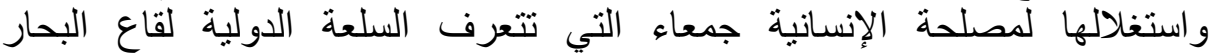

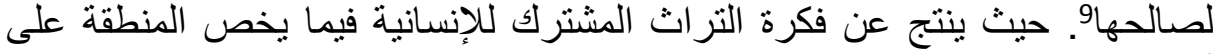
-عدم ادعاء السيادة أو ممارستها على أب جزء من المنطقة أو مو اردهامان.

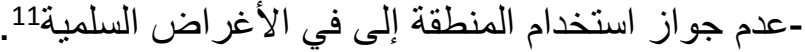

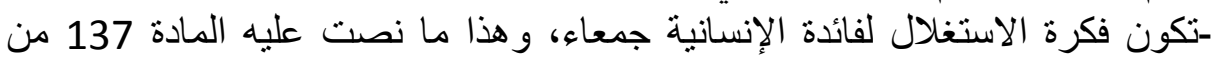
معاهدة 1982 لقانون البحار 12.

\section{ثانيا: السلطة الدولية لقاع البحار}

السلطة هي منظمة دولية مستقلة أنشئت بموجب الاتفاقية والاتفاق المتعلق بتنفيذ الجزء الحادي عشر من اتفاقية الأمم المتحدة لقانون البحار سنة 1994 (اتفاق عام ${ }^{13}(1994$ و السلطة هي المنطقة التي تقوم من خلالها الأطر اف في الاتفاقية، ووفقا للفرع الحادي الإي

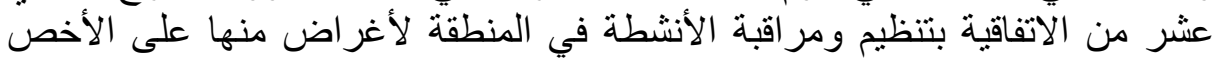

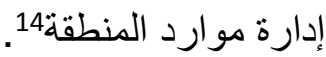
وبموجب اتفاقية الأمم المتحدة لقانون البحار لا يجوز المبن التنقيب عن معادن قاع البحار واستخلالها في المنطقة إلا بموجب الفي عقد مبرم مع الهيئة الدولية لقاع البحار لإن 15. 


\section{المطلب الثاني: المؤثرات السلبية على الحياة البحرية}

منحت السلطة الدولية لقاع البحار مجموعة من التراخيص لعدد معين من الثركات التهات

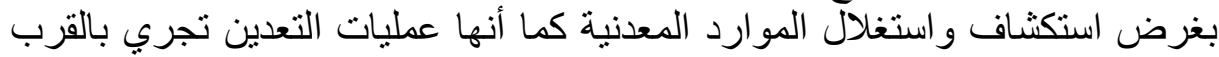

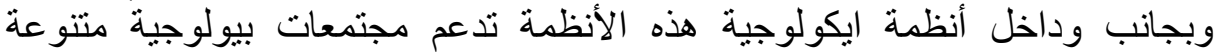

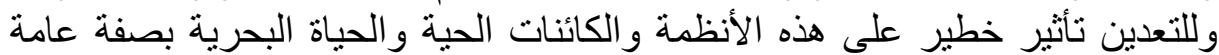

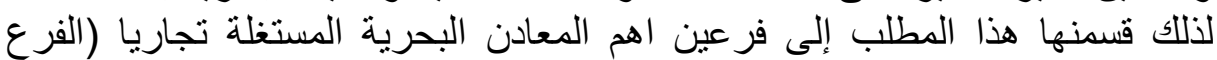

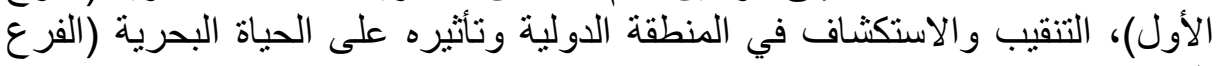

\section{الفرع الأول: أهم المعادن البحرية المستخلة تجاريا}

تحتوي المنطقة الدولية على ملايين الأطنان من المعادن الخام الثمينة ويركز الاهنمام

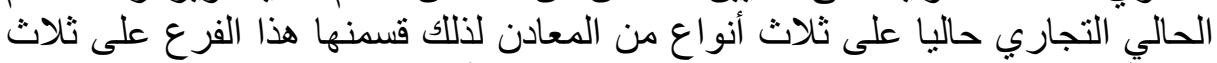

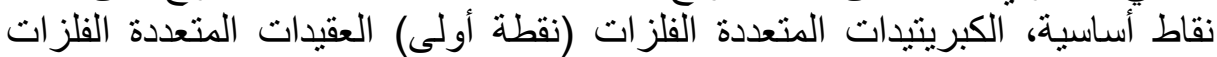
(نقطة ثانية) قشور المنغنيز الحديدي الغنية بالكوبالت (نقطة ثالثة).

أولا: الكبريتيدات المتعددة القلزات:

تشكلت هذه الترسبات عبر آلاف السنين من خلال النشاط الحراري المائي عندما

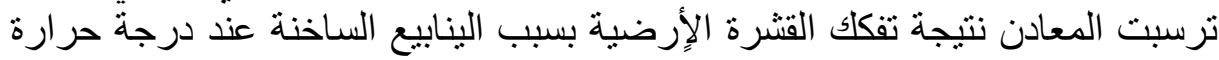

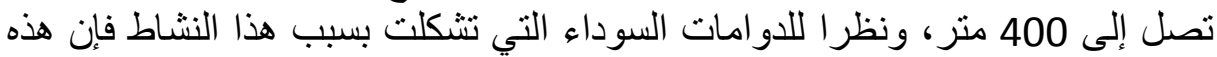

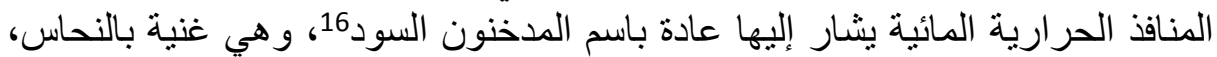

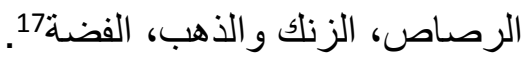

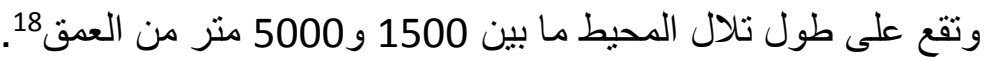

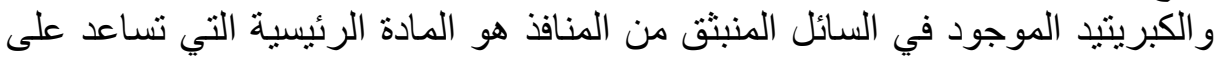

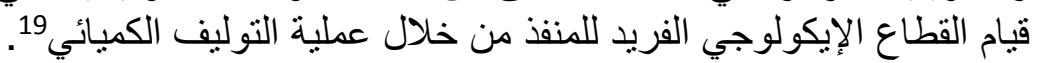

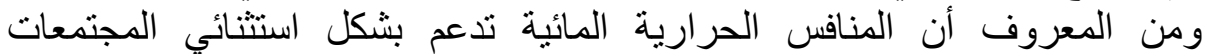

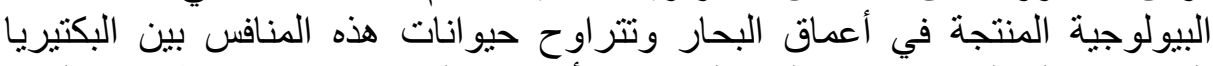

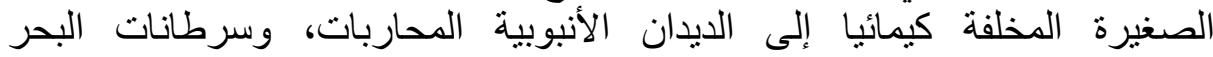

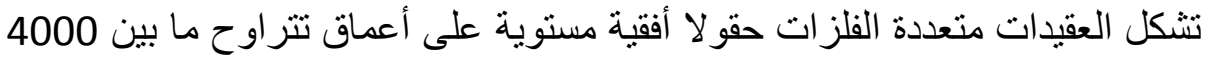

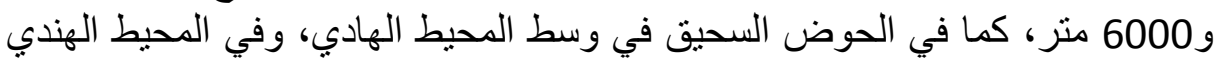

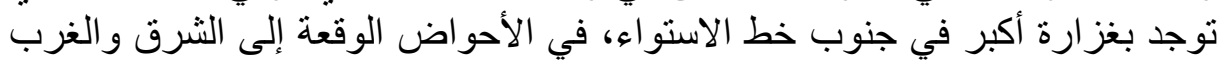
من حيد الدرجة تسعين وتعتبر العقيدات المتعددة الفلزات التي توجد بوفرة في السهول السحيقة بالمنطقة، مصدرا محتملا للنحاس و النيكل، و المنغنيز ، و الكئن الكوبالت22. 
هي الرواسب الهيدروكسيدية الأكسيدية من الحديد والمنغنيز الغنية بالكوبالت التي

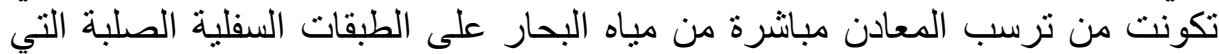

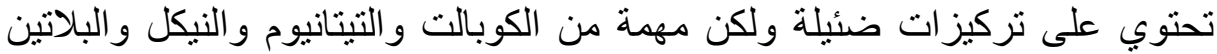

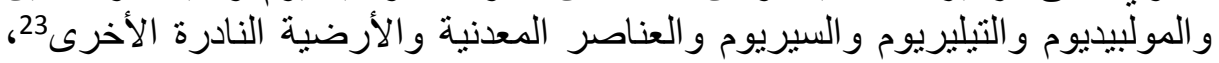
وعقد المنغنيز تتواجد بكميات كبيرة، وتحوي مكونات مغنيزية بنسب تجائ توارية وهي عقد مسودة ومستديرة حجمها في مثل قبضة اليد وتنمو كأغلفة متتابعة فوق الصخور

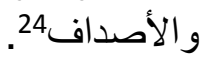

\section{الفرع الثاني: التنقيب والاستكشاف في المنطقة الدولية وتأثيره على الحياة البحرية}

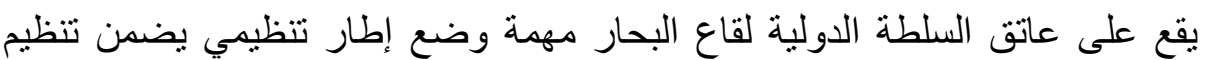

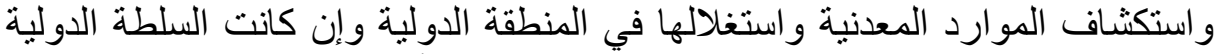

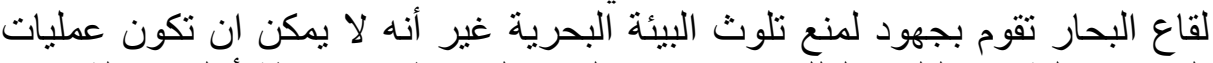

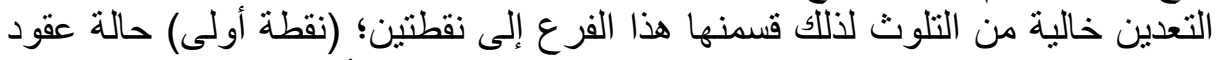

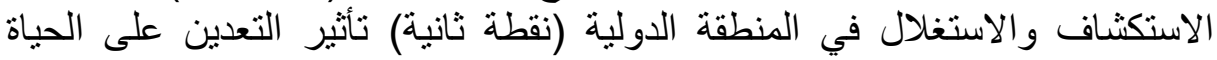

\section{أولا: حالة عقود الاستكشاف والاستغلال في المنطقة الدولية}

تتألف مدونة التعدين حاليا من ثناث مجموعات من الأنظمة تغطي أعمال التنقيب

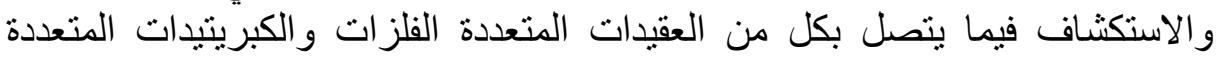

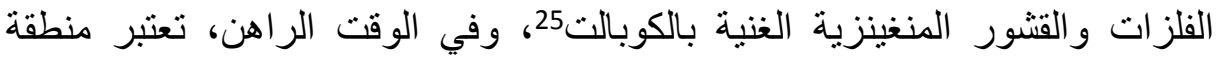

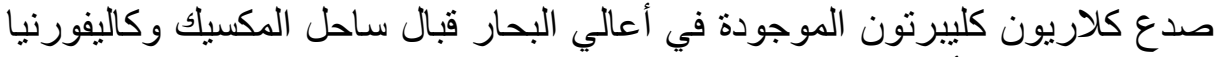

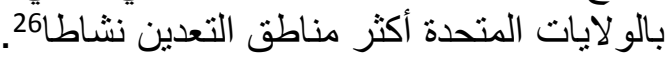

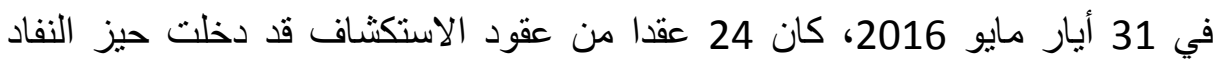

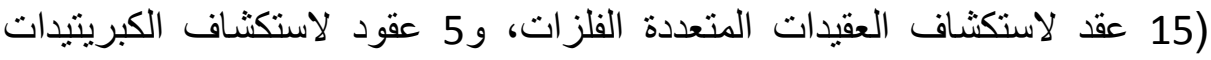

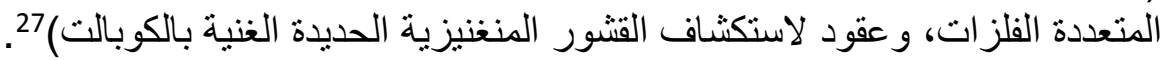
- حيث وقع عقد لاستكثاف العقيدات المتعددة الفلزات مع شركة ( Gook Island

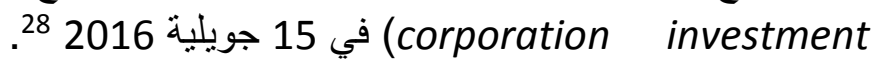
-كما وقع عقد لاستكثاف الكبريتيدات المتعددة الفلزات مع الفئ حكومة الهند في 2015 سبتمبر 2016 ووقع عقد لاستكثاف العقيدات المتعددة الفلزات مع شعندات شركة منمثالز

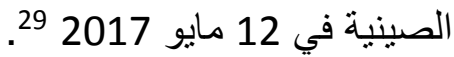

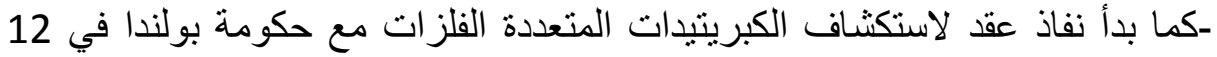

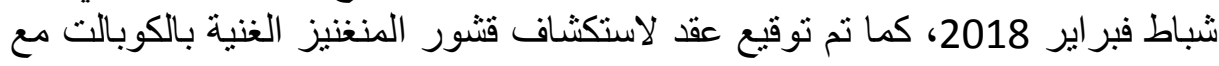

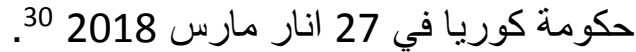
-وييلغ مدة كل عقد يتعلق بخطة عمل للاستكثاف في المنطقة 15 عاما 31 عارس 
ثانيا: تأثير التعدين على الحياة البحرية

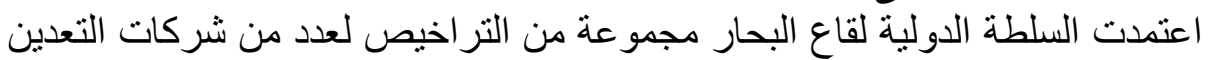

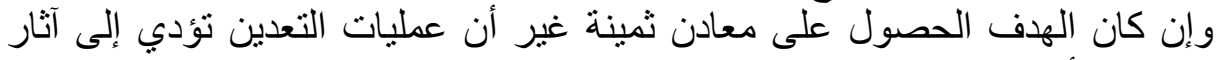
جد سلبية أهمها الهدا ـالتدمير التثامل و على المدى البعيد للأوساط البيئية جراء عملية التعدين التي تتسبب

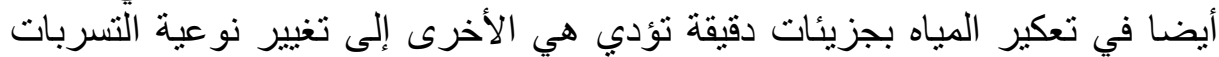

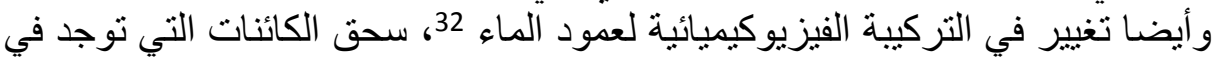

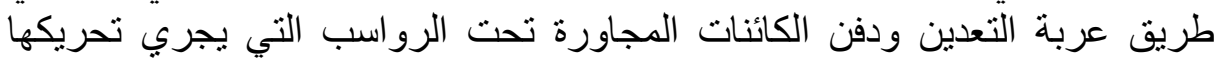

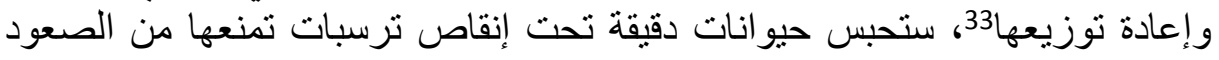

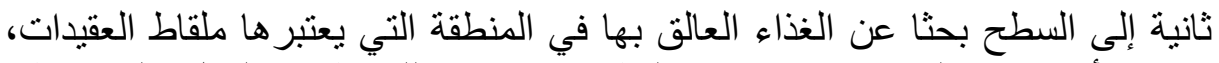

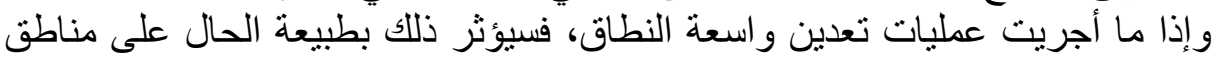

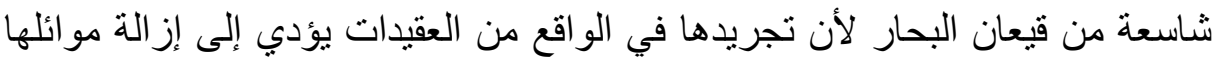

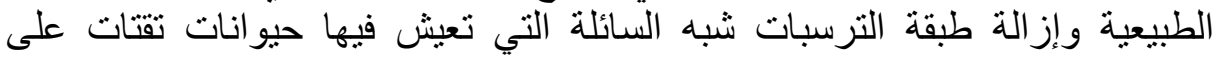

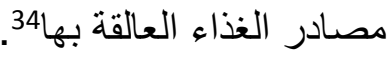

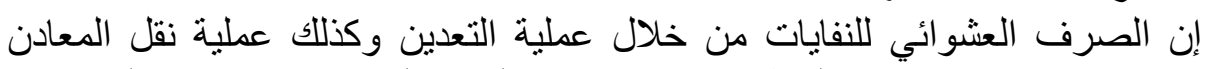

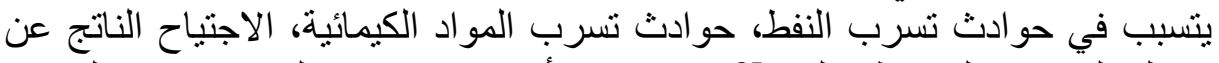

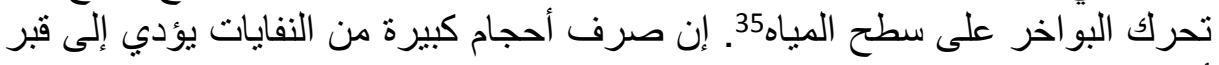
أو دفن الكائنات الحية القليلة الحركة وخفض الحئ في نسبة المخزون الغذائي للكائنات

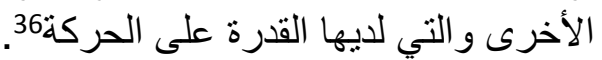

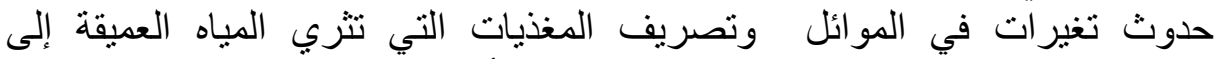

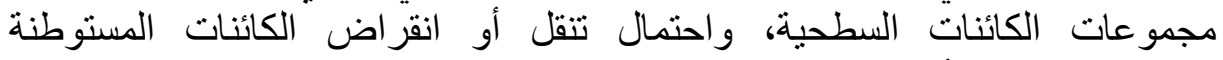

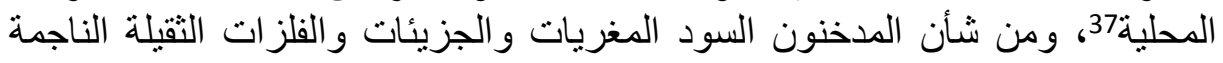

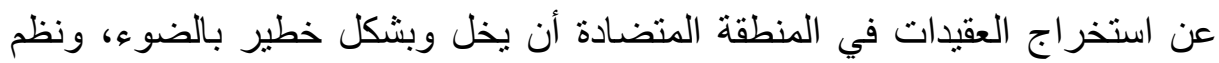

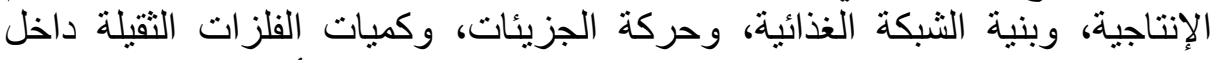

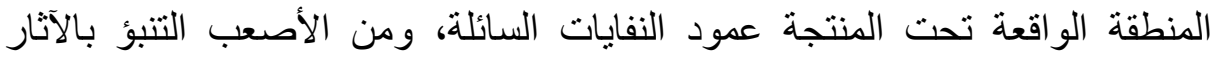

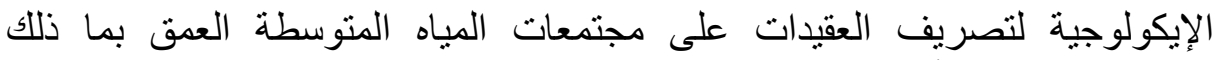
مجتمعات منظمة الأكسجين الدنياندان.

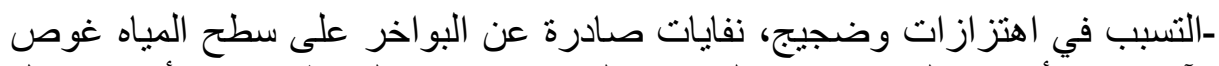

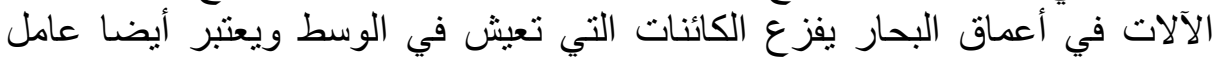

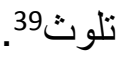

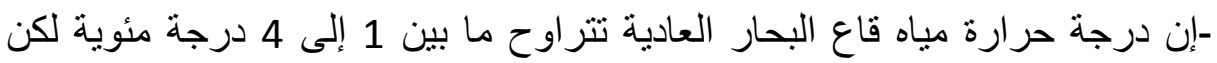

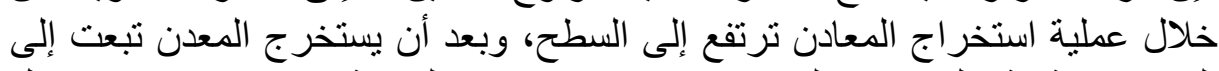

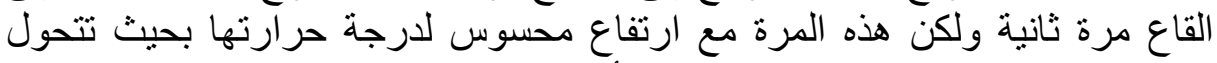

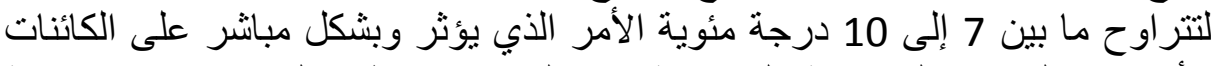

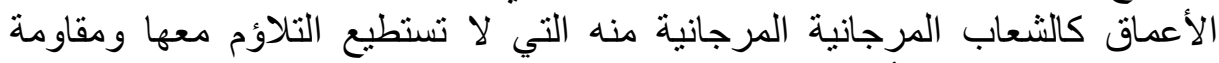
التغيير المفاجئ لهذه الأخيرة النئ.

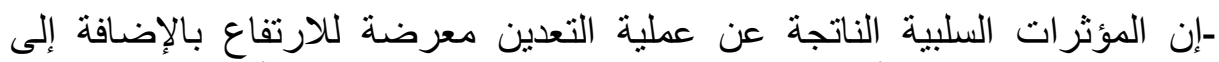

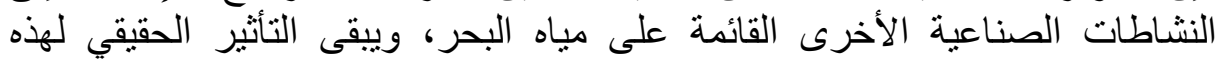
العوامل غير مكتمل المعطيات4 الأنري. 


\section{خـاتـــــة}

بالنسبة لشركات التعدين تعد قاع البحار والمحيطات خارج الولاية الوطنية منجم

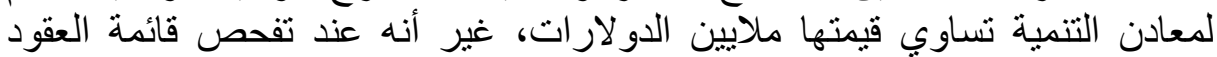

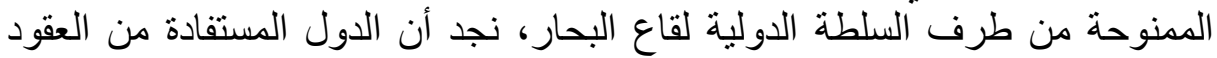

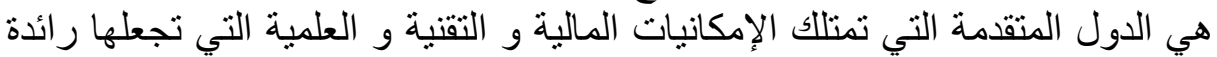

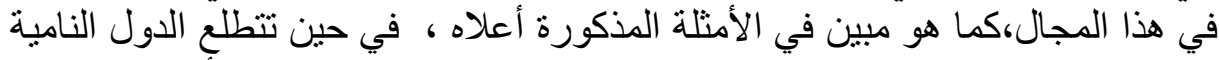

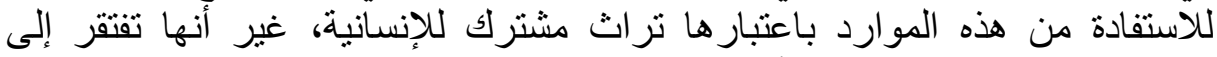

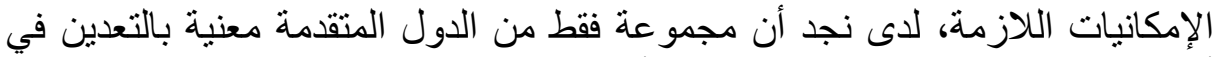

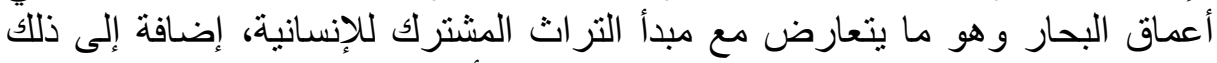

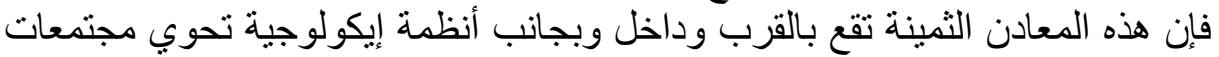

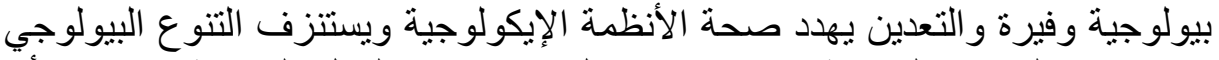

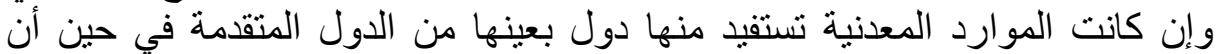
آثار ها المدمرة تعود بالسلب على العى كافة الإنسانية. لذلك نقتر ح ما يلي:

1-وقف عمليات التعدين في المناطق الحساسة كالفتحات الحر ارية المائية.

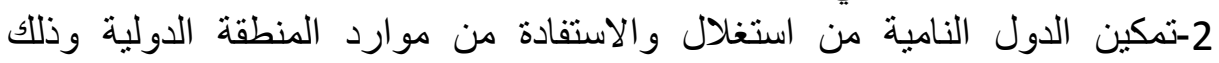

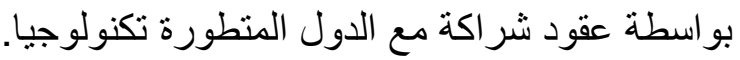

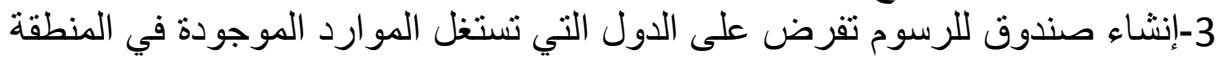

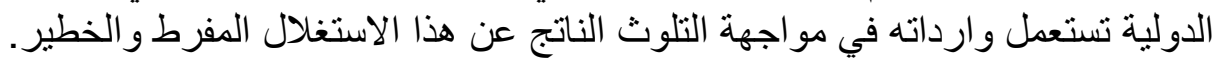

1-تقرير الأمين العام للأمم المتحدة، الدورة التاسعة والخمسون، البند (أ) من القائمة الأولية،

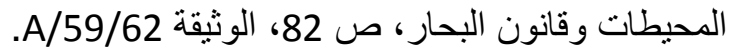

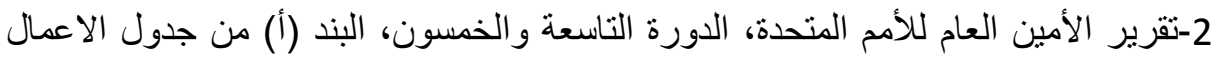

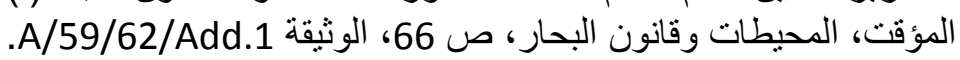

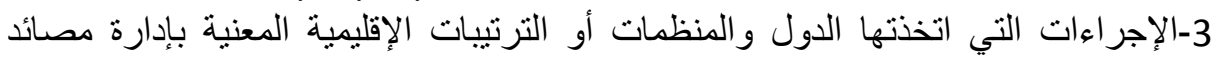

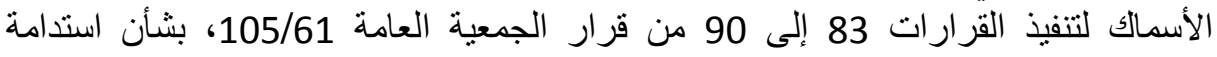

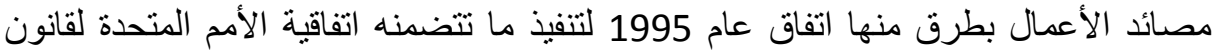

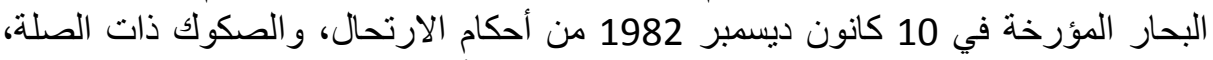

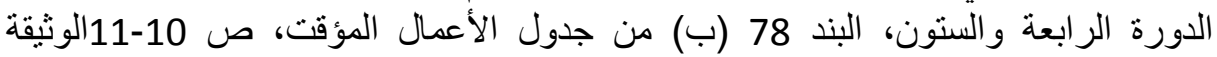
A/64/305.

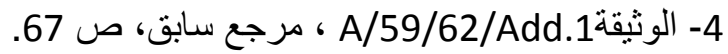

5-الوثيقة 4/59/62،

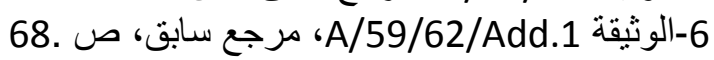

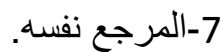

8-رضاء رجب عبد العطيف محمود، الحماية الدولية للبيئة البحرية من التلوث الفوري دار

النهضة العربية، القاهرة، 2019، ص صناء 136.

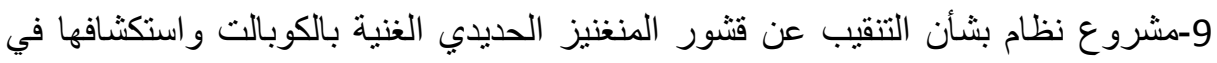

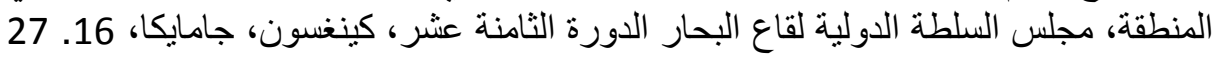
تموزا يوليه 2012، ص 2، الوثيقة ISBA/18/C/WP.1. 
10-إبر اهيم محمد الدمغة، أحكام القانون الدولي لقاع البحار و المحيطات وباطن أرضها خارج

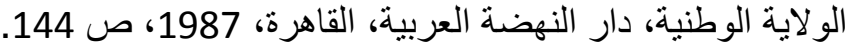

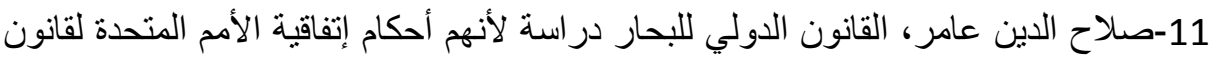

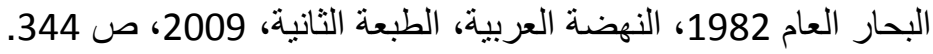
12-محمد سعادي، سيادة الدولة على البحر في القانون الدولي العام، دار الجامعة الجديدة،

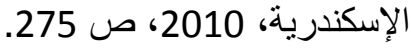

13-نقرير الأمين العام للسلطة الدولية لقاع البحار بموجب الفقرة 4 من المادة 166 من إتفاقية

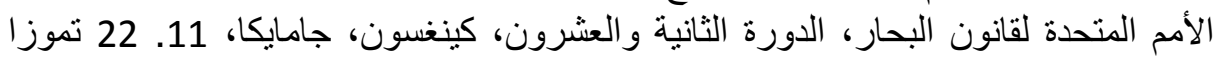

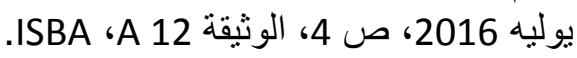

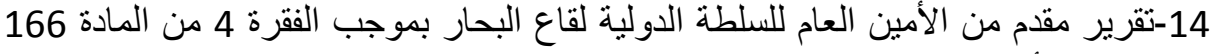

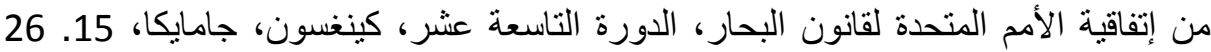

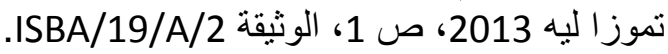
15-ما يكل لودج، الهيئة الدولية لقاع الوثة البحار واستخر اج المعادان من قاع البحر العميق الوقائع،

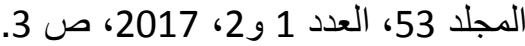

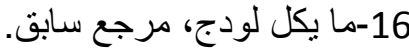

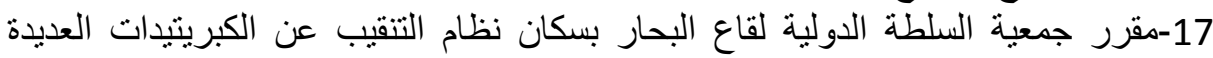

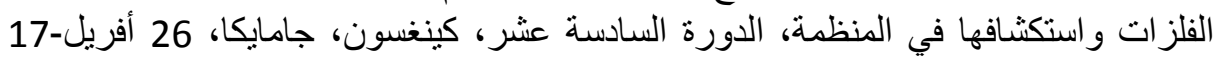

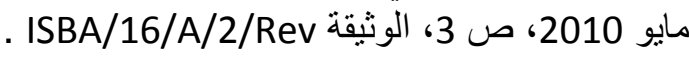
18-KEUIN TROCH, Exploiter les ressources minière sous-marins 24/02/2017, $14 \mathrm{~h} 30$ disponible en ligne: https//trous. Hypotheses.org.116.

19-تقرير الأمين العام للأمم المتحدة، الدورة الثامنة والخمسون، البند 53 من القائمة الأولية،

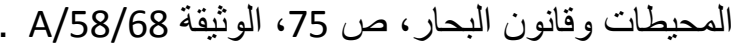

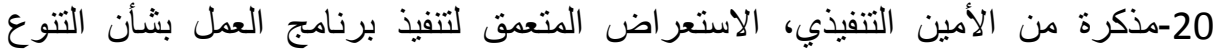

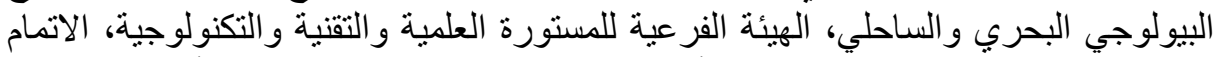

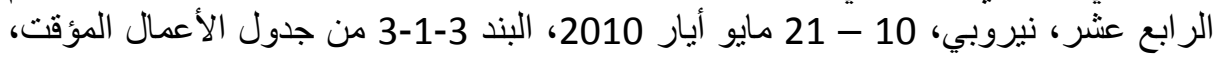

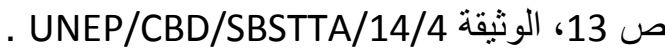

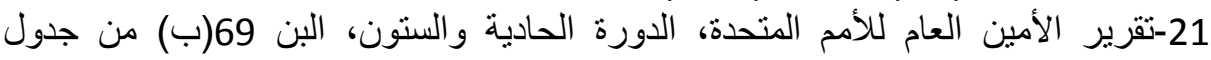

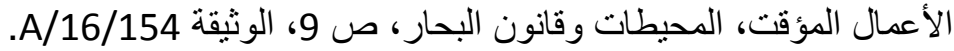

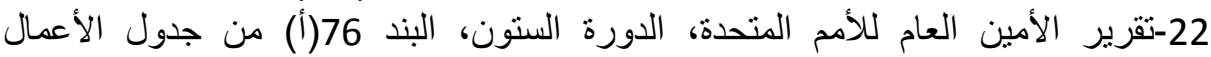

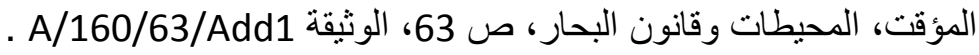

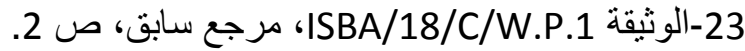
24-جودة حسين جودة، جغرافيا البحار والمحيطات الطبيعية والحيوية، دار المعرفة

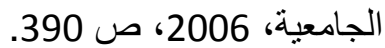

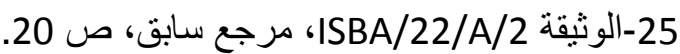

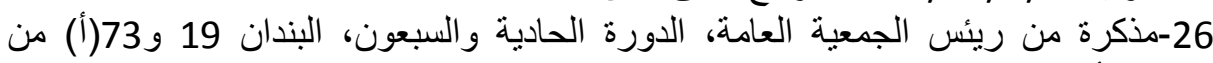

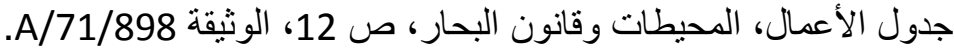

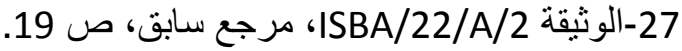
28-Eile Jarmache, $<<$ la législation internationale encadrent l'accès aux ressources minérales marines $\gg>$, Annales des Mines- Responsabilité et environnement 2017/1 ( $\left.N^{\circ} 85\right)$, p 55-61.

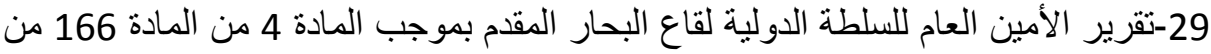

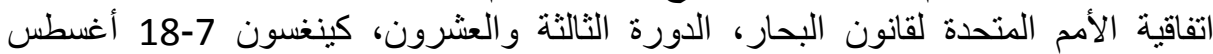




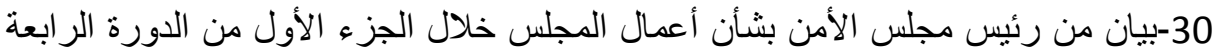

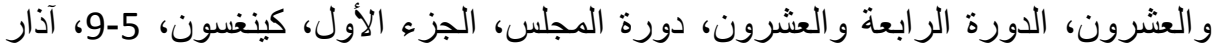

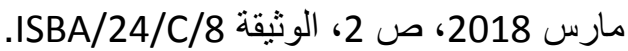

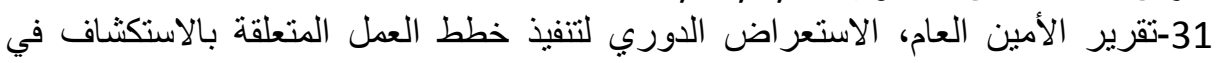

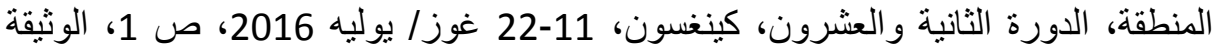
.ISBA $/ 22 / C / 7$

32 -Pierre-Marie Sarradin et la., $<<$ Les impacts environnementaux de l'exploitation minière des fonds marins: un état des lieux des connaissances $\gg$, Annales des Mines- Responsabilité et environnement 2017/1 ( $\left.N^{\circ} 85\right)$, p 30-34.

33-تقرير الأمين العام للأمم المتحدة، الدورة الثانية والستون، البند 79(أ) من جدول الأعمال

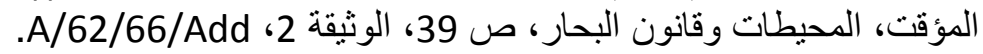

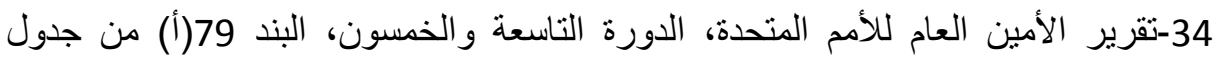
الأعمال المؤقت، المحيطات وقانون البحار، ص 32 32، الوثيقة 1، A/62/66/Add.

35-Rob timch, Sybille ran den home, Report on Palcy option and Associated valuation and appraisal needs and maths ods deliverable $9.5,20$ septembre 2016, p 28.

36-J.Dyment, F. Allier, N.Le Bris, O. Rouxel, P.-M. Sarradin, S. Lamer, C. Coumert, M. Morineaux, J. Tourille (coord.), 2014. Les impacts environnementaux de l'expoitation des ressources minérales marines profondes. Expertise scientifique collective, Synthèse du rapport, CNRS-ifermer, 110p. environ

37-مقرر معتمد من مؤتمر الأطراف في اتفاقية التنوع البيولوجي، مؤتمر الأطراف في الأني

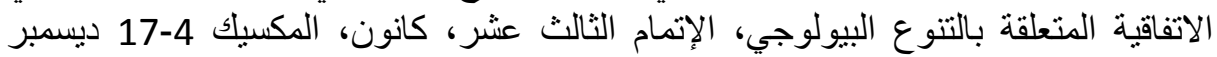

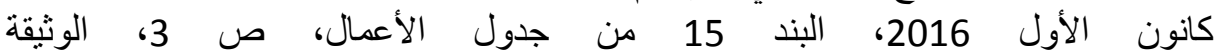
.CBD/Cop/DEC/XIII/11

38-تقرير الأمين العام، الدورة الستون، البند 76/(أ) من جدول الأعمال المؤقت، الدحيطات

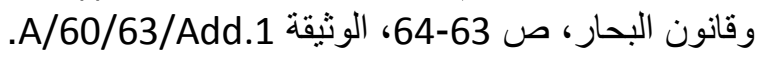

39- Pierre-Marie Sarradin, op. cit, p 33.

40- J.Dyment, F. Allier, et autres, op. cit, p 67.

41- Pierre-Marie Sarradin, op. cit , p 33. 